\title{
MODEL OF FORCED TURBULENCE FOR PULSING FLOW
}

\author{
Vasyl DMYTRIV \\ Lviv Polytechnic National University, Institute of Engineering Mechanics and Transport, Lviv, Ukraine \\ Dmytriv_V@ukr.net
}

\begin{abstract}
The article deals with fluid motion along an infinite hose. Taking into consideration the Stokes equation, the tangential friction stresses according to Newton and the Reynolds equation, the differential equation of the velocity change in radius is developed taking into account the pulsating component of the friction forces in the turbulent flow. Turbulence is defined as the impulse component of a flow, characterized by a pressure drop along a dynamic length of flow, a frequency response, and an oscillation amplitude of the pressure drop of pulse (which is given by the time equation of the oscillation). The velocity distribution along the radius of the hose in the time interval of one second was modelled for pressure drops in the range from 6000 to $18000 \mathrm{~Pa}$ and the amount of transported medium in the range from $1.667 \cdot 10^{-5}$ to $6.667 \cdot 10^{-5} \mathrm{~m}^{3}$, which corresponded to the length of pulse plug. The dynamic viscosity of the medium (milk) of $1.79 \cdot 10^{-3} \mathrm{~Pa} \cdot \mathrm{s}$ and its density of 10273 $\mathrm{N} \cdot \mathrm{s}^{2} / \mathrm{m}^{4}$ were accounted at the simulation. The developed analytical dependence of the velocity of the forced turbulence of the pulsating flow allows to calculate the absolute value of the velocity at a given point of crosssection of the pipeline, and characterizes the physical process of flow of Newtonian fluids and gases in the pipeline.
\end{abstract}

Keywords: forced turbulence, velocity of pulsating flow, dynamic length, pressure drop, Karman constant.

\section{INTRODUCTION}

In the problems of automation of complicated technological processes that ensure the adaptation of systems of different origin, the problem of analytical identification of process parameters arises. Such systems contain processes that are influenced by several factors simultaneously and lead to changes in the process characteristics. In particular, the pulsating fluid flow caused by the equipment operating mode is considered in the work, namely impulse change of pressure in technological and technical pipelines of automation systems, milk transport by milking machine and a number of other technical systems operating at critical modes with high frequency of change. The pulsating mode of motion creates "forced" turbulence. To determine the quantitative characteristic (production or effectiveness) and qualitative process parameters (flow mode, intensity), it is necessary to have a characteristic of velocity distribution. A simpler case - the pipeline is rigid and the more complicated process - the pipeline is flexible.

Therefore, the elastic pipeline - the milk hose of the milking machine is chosen, which is relevant to adapt the work of the technical system to the physiological requirements of the cow's milk ejection.

The task of turbulent flows research including forced turbulence based on external disturbances is complex, and often researchers use a number of assumptions and use numerical methods to solve them.
Authors T. S. Lee, X. Liu, G. C. Li and H. T. Low used the numerical simulations by the finite-volume method of second-order based on a disordered nonorthogonal net for laminar sinusoidal pulsating flow in a tube with smooth single narrowing. At that they studied the effects of the Reynolds number, the Womersley number, the ripple amplitude, the narrowing factor, the narrowing length and fluid flow in the narrowed tube [1].

To develop analytical models, both laminar and turbulent flows, it is necessary to analyse and mathematically describe the process of transportation of the test medium - single-phase or two-phase Newtonian gas-liquid medium. The stable flow in the tube, determined by the Reynolds number, has been sufficiently studied today and also the scenario of its study has been developed [2, 3, 4]. In contrast to the steady flow, the amplitude and frequency of the flow pulsations expressed by the Womersley number must be determined in addition to the Reynolds number. However, the analysis of the above studies showed that these are experimental studies only that are not described by regression dependencies and its reproducibility is a difficult task.

In mechanics of biological fluid authors studied for which the Reynolds and Womersley numbers and ripple amplitudes the flow are transformed into turbulent [5].

Also, during studies of forced pulsating flow, the time shift in the flow response to the applied oscillation amplitude was determined [6].

Some researchers for the analysis of pulsating turbulent flows select the Fourier series to the 
average values of the average and turbulent velocities, or other parameters that characterize the flow. Similarity parameters for the correlation of studied results were defined to comprehend, or at least to classify, how the amplitude and phase of these Fourier series depend on the time-averaged flow rate, Reynolds number and pulse rate. And also the time-averaged volumetric velocity, pipe line diameter, kinematic viscosity and frequency of the forcing are indicated respectively [7]. Stokes laminar length serves as an etalon for comparison. Therefore, it is used for comparison with the laminar pulsating flow and as a value for scaling.

Previous studies have shown that many values were scaled with the Stokes-Reynolds number, which is equivalent to the normalized angular frequency $[8,9,10]$.

By definition the Stokes length is a purely laminar concept. Therefore, in order to account the diffusivity of impulse in turbulent flows, Scotti A. and Piomelli U. introduced the concept of Stokes turbulent length [11].

In particular, He S. and Seddighi M. used direct numerical simulation (DNS) in order to model the accelerated flow in a channel with a stepwise change in flow velocity and is not a pulsating flow [12]. These authors also showed that the ratio of the initial to the final Reynolds numbers characterizes the behavior of the transition stream. But the temporal data from pulsating flows are sparse, where the large eddy simulation (LES) given in [11] and the DNS in [13] are two exceptions. Comparison of the turbulence response predicted by the LES during the phase of eddy acceleration with the results of the linear accelerated DNS [14] shows a high similarity between the two cases. The initial turbulence response consists of velocities varying along the flow in conditions increase of flow strip, and subsequently after the initial delay, turbulence increases rapidly as a result of the formation and confluence of turbulent flow areas [15].

Qualitatively, such a development of turbulence in a pulsating flow can be deduced from the fundamental regime by the Fourier series by calculating the phase difference between the averaged parameters of the forced turbulent pulse oscillations. But all analyzed researchers did not investigate the nature of velocity distribution and velocity as a major parameter. They also practically did not analytically describe the dependence of velocity parameters on the factors that influence the formation of forced turbulence.

\section{ANALYTICAL MODEL OF FORCED TURBULENCE}

\subsection{Theoretical assumptions for forced turbulence beginning}

One of the main parameters affecting the flow dynamics is the velocity, which depends on many factors, namely the intensity of the flow, the pressure drop in the hose, the linear dimensions of the hose, the characteristics of the medium, the frequency and amplitude of external disturbances in the flow of the medium $[16,17]$.

Let me consider a model of the state of a moving medium by using functions that determine the velocity distribution of a liquid mixture in the longitudinal and cross sections of the hose of $v=f(X, Y, Z, t)$ and two dynamic parameters, such as $p=f(X, Y, Z, t)$ pressure and the density of the liquid mixture of $\rho_{c y s}=f(X, Y, Z, t)$. As it is known, all dynamic parameters are determined by the values of two of them by means of the equation of state of the medium. Therefore, the motion of the liquid mixture was determined completely with specifying of five quantities: three components of $v$ velocity, $p$ pressure and $\rho_{c y M}$ density.

These parameters are functions of $X, Y, Z$ coordinates and $t$ time. Let us suppose that $v=f(X, Y, Z, t)$ is the velocity of the liquid mixture at each point of $X, Y, Z$ of space at time of $t$, (it refers to specific points of space, not to fluid particles that move at time in space). We also take such assumptions to the parameters of $p$ and $\rho_{p}$. With taking into account that the liquid mixture consists of liquid and air, and the condition of the air velocity is higher than the fluid velocity, the density of this such mixture will vary along the length of the hose (along the $\mathrm{X}$ axis), and thus the two-phase mixture can be considered as non-compressed.

\subsection{Development of the analytical model of turbulent motion}

The fluid motion along an endless hose is considered. Axis system is positioned so that the $X$ axis is directed along the axis of the hose. The equality of $V=\omega=0$ are assumed, and the velocity component depends only on $R$ radius. The pressure is considered constant throughout the space, respectively the laminar sublayer is in the immediate vicinity of the wall. For this case, the Stokes equation are used in the following form

$$
\frac{d}{d R} \eta \cdot \frac{d U_{T}}{d R}=0
$$

after integrating of formula 1 , we will get

$$
\left[\mu \cdot \frac{d U_{T}}{d R}\right]_{R-r_{0}}=C=\tau_{\omega},
$$

where $\tau_{\omega}$ - tangential stress of friction by Newton.

After the second integration, value of the $U_{T}$ speed will be

$$
U_{T}=\frac{\tau_{\omega}}{\mu} \cdot R+C_{1}
$$

The $C_{1}$ constant of integration is determined from the initial conditions, for $R=r_{0}, U_{T}=0$, on the wall the velocity will be zero and, accordingly, for the laminar flow velocities are linearly distributed: 


$$
U_{T}=\frac{\tau_{\omega}}{\mu} \cdot R
$$

For the profile of velocities under the turbulent mode of motion the Reynolds equation [18] was applied, respectively after assuming that all flow parameters are independent of the $X$ ordinate. With the appropriate corrections, we obtain the following equation:

$$
\frac{d}{d R}\left[\mu \cdot \frac{d U_{T}}{d R}+\left(-\rho_{p} \cdot \overline{U_{T} \cdot V}\right)\right]=0,
$$

where $U_{T}$ - pulsating velocity along the $X$ axis;

$V$ - pulsating velocity in direction of $R$ radius; or

$$
\frac{d}{d R}\left(\mu \cdot \frac{d U_{T}}{d R}+\tau\right)=0,
$$

where $\tau=-\rho_{p} \cdot \overline{U_{T} \cdot V}$

After integration over $R$ we get

$$
\mu \cdot \frac{d U_{T}}{d R}+\tau=C_{2} .
$$

Under boundary conditions, when $R=r_{0} \rightarrow \tau=0$ , the permanent of integration will be:

$$
C_{2}=\tau_{\omega}=\left.\mu \cdot \frac{d U_{T}}{d R}\right|_{R-r_{0}},
$$

accordingly, the equation of (7) will take the form of:

$$
\mu \cdot \frac{d U_{T}}{d R}+\tau=\tau_{\omega} .
$$

In (8) equation, the stress of viscous friction will be quite large in the immediate vicinity to the hose wall. The stress of turbulent friction near the wall will be small. To study the velocity profile in the turbulent area, let us consider the case when the following condition is satisfied:

$$
\mu \cdot \frac{d U_{T}}{d R}<<<\tau .
$$

Then with taking into account of (8) equation we will get:

$$
\tau=\tau_{\omega}=-\rho_{p} \cdot \overline{U_{T} \cdot V}
$$

By analogy with viscous friction, the turbulent stress can be written in the form of:

$$
\tau=A \cdot \frac{d U_{T}}{d R}=\rho_{p} \cdot \frac{A}{\rho_{p}} \cdot \frac{d U_{T}}{d R}=\rho_{p} \cdot K \cdot \frac{d U_{T}}{d R},
$$

where $A$ and $K$ - analogues of absolute and kinetic coefficients of turbulent transfer.

We reduce the equation of (11) to a form that is convenient for practical use, assuming, by analogy with viscous friction, that the value of turbulent friction depends on the $\frac{d U_{T}}{d R}$ velocity gradient. It follows from equations of (8) and (11) that the value of turbulent friction is a function of the $R$ distance from the wall of the hose and depends on the $\rho_{p}$ density of liquid. Let us assume that

$$
\tau^{\prime}=\rho_{p}{ }^{X 1} \cdot\left(-\frac{d U_{T}}{d R}\right)^{X 2} \cdot l_{p r}^{X 3}
$$

where $l_{p r}$ - the length of interfusion.

After applying the method of substitution of dimensions, we will get the following expression

$$
\left[\frac{\mathrm{kg} \cdot \mathrm{m}}{\mathrm{s}^{2} \cdot \mathrm{m}^{2}}\right]=\left[\frac{\mathrm{kg}}{\mathrm{m}^{3}}\right]^{\mathrm{X} 1} \cdot\left[-\frac{1}{\mathrm{~s}}\right]^{\mathrm{X} 2} \cdot[\mathrm{m}]^{\mathrm{X} 3} \text {. }
$$

Accordingly, we obtain a system of equations

$$
\left\{\begin{array}{l}
\mathrm{X} 1=1 \rightarrow \text { for } \mathrm{kg} \\
-1=-3 \cdot \mathrm{X} 1+\mathrm{X} 3 \rightarrow \text { for } \mathrm{m} \\
-2=-\mathrm{X} 2 \rightarrow \text { for } \mathrm{s}
\end{array}\right.
$$

The solution of (14) equation gives the following values of powers: $\mathrm{X} 1=1 ; \mathrm{X} 2=2 ; \mathrm{X} 3=2$.

Accordingly, the equation of the turbulent stress of friction will have the form

$$
\tau^{\prime}=\tau_{\omega}=\rho_{p} \cdot l_{p r} 2\left(\frac{d U_{T}}{d R}\right)^{2} .
$$

It is convenient to replace the transference length with an expression of $l_{p r}=K a \cdot R$.

Therefore, due to forced turbulence, which is characteristic for the pulsating nature of the transport of the liquid-air mixture, between the layers there will be both turbulent and laminar stress of friction. In view of (15) equation, the stress of friction between the layers is written as follows:

$$
\tau=\mu \cdot \frac{d U_{T}}{d R}+\rho_{p} \cdot(K a \cdot R)^{2} \cdot\left(\frac{d U_{T}}{d R}\right)^{2} .
$$

where $\mu \cdot \frac{d U_{T}}{d R}$ - viscous friction by I. Newton, which corresponds to the forces of friction in the laminar flow;

$$
\rho_{p} \cdot(K a \cdot R)^{2} \cdot\left(\frac{d U_{T}}{d R}\right)^{2}-\quad \text { the pulsating }
$$

component of the friction forces in a turbulent flow.

After solving of (16) equation with regard to $d U_{T} / d R$, we will get:

$$
\frac{d U_{T}}{d R}=\frac{-\mu \pm \sqrt{\mu^{2}+4 \cdot \rho_{p} \cdot K a^{2} \cdot R^{2} \cdot \tau}}{2 \cdot \rho_{p} \cdot K a^{2} \cdot R^{2}} .
$$

In view of $\tau=\rho_{p} \cdot V_{*}^{2}$ and integrating over $R$, we will get:

$$
\begin{aligned}
& U_{T}=\frac{\mu}{2 \cdot \rho_{p} \cdot K a^{2}} \cdot \frac{1}{R} \mp \frac{V_{+}}{K a \cdot R} \times \\
& \times \sqrt{\frac{\mu^{2}}{4 \cdot \rho_{p}^{2} \cdot K a^{2} \cdot V_{+}^{2}}+R^{2} \pm} \\
& \pm \frac{V_{+}}{K a} \cdot \ln \left(R+\sqrt{\frac{\mu^{2}}{4 \cdot \rho_{p}^{2} \cdot K a^{2} \cdot V_{+}^{2}}+R^{2}}\right)+C
\end{aligned}
$$

or

$$
U_{T}=\frac{\mu}{2 \cdot \rho_{p} \cdot K a^{2} \cdot R} \mp \frac{V_{*}}{K a \cdot R} \times
$$




$$
\begin{aligned}
& \times \sqrt{\frac{\mu^{2}}{4 \cdot \rho_{p}^{2} \cdot K a^{2} \cdot V_{*}^{2}}+R^{2} \pm \frac{V_{*}}{K a} \times} \\
& \times \operatorname{asinh}\left(R \cdot \sqrt{\frac{4 \cdot \rho_{p}^{2} \cdot K a^{2} \cdot V_{*}^{2}}{\mu^{2}}}\right)+C,
\end{aligned}
$$

where $V_{*}$ - the dynamic velocity on the wall of the hose;

$R$ - the distance from the wall of the hose to the point of determining the velocity;

$C$ - the constant of integration.

The $C$ constant of integration is determined from the initial conditions. $U_{T}=U_{C T}=\alpha \cdot V_{*}$ is on the wall of the hose at $R=0$. After substituting of $R=0$ into the denominator of the items of (18) equation, we will get that they go to infinity, which contradicts the initial conditions. Therefore, we will get the following value for the $C$ constant of integration:

$$
C=\alpha \cdot V_{*} \text {. }
$$

After substitute of $C$ into (19) equation, we will get the following dependence for determining the turbulent velocity distribution:

$$
\begin{gathered}
U_{T}=\frac{\mu}{2 \cdot \rho_{p} \cdot K a^{2} \cdot R} \mp \frac{V_{*}}{K a \cdot R} \times \\
\times \sqrt{\frac{\mu^{2}}{4 \cdot \rho_{p}^{2} \cdot K a^{2} \cdot V_{*}^{2}}+R^{2} \pm \frac{V_{*}}{K a} \times} \\
\times \operatorname{asinh}\left(R \cdot \sqrt{\frac{4 \cdot \rho_{p} \cdot K a^{2} \cdot V_{*}^{2}}{\mu^{2}}}\right)+\alpha \cdot V_{*} .
\end{gathered}
$$

The thickness of the boundary layer can be determined from equation of [16]:

$$
\delta_{c}=\varepsilon \cdot \frac{\eta}{\sqrt{\rho_{p} \cdot \tau}}=\alpha \cdot \frac{v}{\sqrt{\frac{\tau}{\rho_{p}}}}=\alpha \cdot \frac{v}{V_{*}}=\alpha \cdot l_{*},
$$

where $\alpha$ - the Landau constant;

$$
\begin{aligned}
& V_{*}=\sqrt{\frac{\tau_{\omega}}{\rho_{p}}}-\text { the dynamic velocity; } \\
& l_{*}=\frac{v}{V_{*}}-\text { the dynamic length. }
\end{aligned}
$$

In the hose the $\pi^{\cdot} r_{\mathrm{o}}^{2} \cdot \Delta p$ moving force acts on the entire cross section of the fluid flow. This force is aimed at overcoming of wall friction. Since the ratio of the friction force to the unit of wall area is $\tau=\rho_{p} \cdot V_{*}^{2}$, the total friction force is $2 \cdot \pi \cdot r_{0} \cdot l_{p r}$. $\rho_{p} \cdot V_{*}^{2}$. Taking into consideration the expressions of forces, we will get:

$$
\frac{\Delta P}{l_{p r}}=\rho_{p} \cdot V_{*}^{2} \cdot \frac{2}{r_{0}} .
$$

Equation of (23) determines in a parametric form the relationship of the velocity of the fluid in the hose with the pressure drop in it ( $V_{*}$ parameter).

From (23) equation it follows that

$$
V_{*}=\sqrt{\frac{\Delta P \cdot r_{0}}{2 \cdot l_{p r} \cdot \rho_{p}}} .
$$

After substitute of (24) equation into (21) equation and change of $R=r_{0}-r_{1}$ and $l_{p r}=Q /\left(2 \cdot \pi \cdot r_{0}{ }^{2}\right)$, we will get:

$$
\begin{aligned}
U_{T} & =\frac{\mu \cdot\left(r_{0}-r_{1}\right)^{-1}}{2 \cdot \rho_{p} \cdot K a^{2}} \mp \frac{\left(r_{0}-r_{1}\right)^{-1}}{K a} \cdot \sqrt{\frac{\Delta p \cdot \pi \cdot r_{0}^{3}}{Q \cdot \rho_{p}}} \times \\
& \times \sqrt{\frac{\mu^{2} \cdot Q}{4 \cdot \pi \cdot \rho_{p} \cdot K a^{2} \cdot \Delta p \cdot r_{0}^{3}}+\left(r_{0}-r_{1}\right)^{2} \pm} \\
& \pm \sqrt{\frac{\Delta p \cdot \pi \cdot r_{0}^{3}}{Q \cdot K a^{2} \cdot \rho_{p}} \cdot \operatorname{asinh}\left(\left(r_{0}-r_{1}\right) \times\right.} \\
& \left.\sqrt{\frac{4 \cdot \rho_{p} \cdot K a^{2} \cdot \Delta p \cdot \pi \cdot r_{0}^{3}}{\mu^{2} \cdot Q}}\right)+\alpha \cdot \sqrt{\frac{\Delta p \cdot \pi \cdot r_{0}^{3}}{\rho_{p} \cdot Q}}
\end{aligned}
$$

where $\Delta P$ - the pressure drop, $\mathrm{Pa}$;

$r_{\mathrm{o}}-$ the radius of hose, $\mathrm{m}$;

$r_{1}$ - the length from hose center to point of velocity determining, $\mathrm{m}$;

$\rho_{p}-$ the density of liquid, $\mathrm{N} \cdot \mathrm{s}^{2} / \mathrm{m}^{4}$;

$l_{p r}$ - the length of the impulse plug of the transport medium in the hose, $\mathrm{m}$;

$Q$ - the quantity of medium corresponding to the length of the impulse plug, $\mathrm{m}^{3}$;

$\mathrm{Ka}$ - the Karman constant;

$\alpha$ - the Landau constant;

$\mu$ - the dynamic viscosity of the liquid, $\mathrm{Pa} \cdot \mathrm{s}$.

The derived equation of (25) is characterized by the velocity distribution in the hose (pipeline) taking into account the laminar boundary layer.

\section{RESULTS OF SIMULATION OF VELOCITY OF PULSING TURBULENT MOTION}

Velocity distribution by hose radius in the time interval of one second was simulated at the conditions of the $\Delta P$ pressure drop and the $Q$ medium quantity of the $\mu$ dynamic viscosity that correspond to the pulse length in the hose of $r_{0}$ radius. To experimental study following parameters were taken: dynamic viscosity of the medium was $\mu=1.79 \cdot 10^{-3} \mathrm{~Pa} \cdot \mathrm{s}$ for milk, milk density was $\rho_{p}=10273 \mathrm{~N} \cdot \mathrm{s}^{2} / \mathrm{m}^{4}$, Landau constant was $\alpha=11.5$, Karman constant was $K a=0.41$, hose radius $r_{0}=0.008 \mathrm{~m}$. The $\Delta p$ pressure losses ranged from 6000 to $18000 \mathrm{~Pa}$, and the $Q$ quantity of milk ranged from $1.667 \cdot 10^{-5} \mathrm{~m}^{3}$ to $6.667 \cdot 10^{-5} \mathrm{~m}^{3}$, which corresponded to the length of the pulse plug.

The results of the simulation in one second interval are shown on Fig. 1-3.

Fig. 1 shows the 3-D models of the velocity distribution over the diameter of the hose in the interval of one second cycle of the impulse plug of the mixture flow.

Fig. 2 and 3 represent in the plane the $\boldsymbol{U}_{\boldsymbol{T}}$ velocity change of the pulsating flow and the $\boldsymbol{U}_{T}$ maximum velocity in $\boldsymbol{t}$ time and over the $\boldsymbol{r}_{\mathbf{0}}$ radius of the hose. 


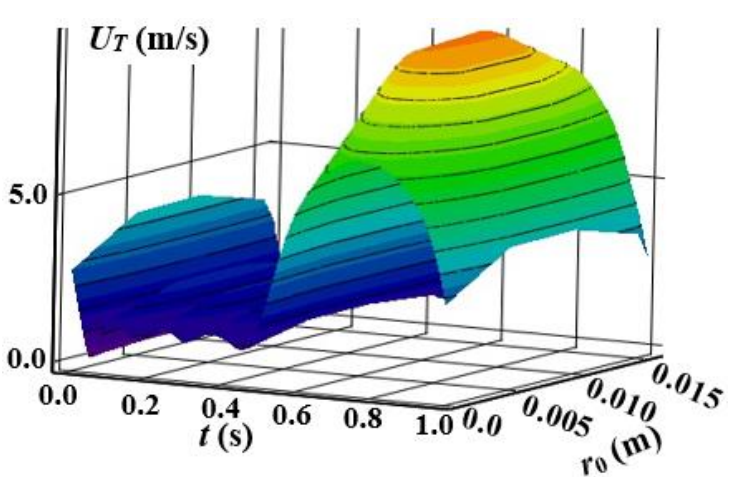

a

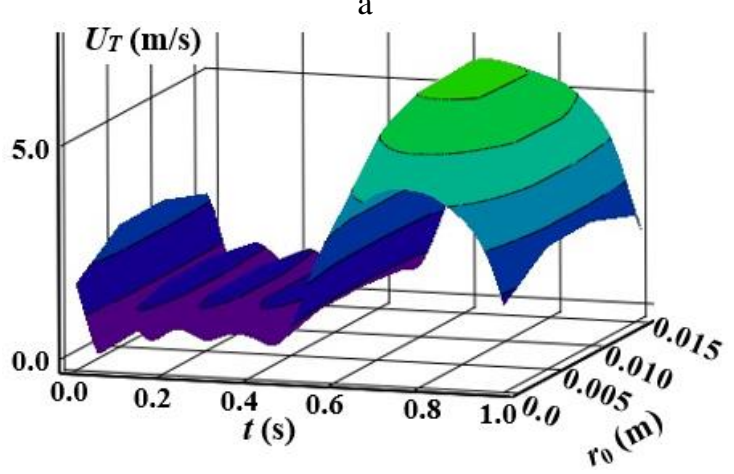

Fig. 1. 3-D model of change of the $\boldsymbol{U}_{T}$ velocity of pulsatory flow over the $\boldsymbol{t}$ time and $\boldsymbol{r}_{\boldsymbol{0}}$ radius by the parameters of $\Delta \boldsymbol{p}$ pressure lost and $\boldsymbol{Q}$ quantity of liquid that corresponds with the length of the impulse plug:

$$
\begin{aligned}
& \text { a }-\Delta \boldsymbol{p}=10 \mathrm{KPa}, \boldsymbol{Q}=1.667 \cdot 10^{-5} \mathrm{~m}^{3} ; \\
& \mathrm{b}-\Delta \boldsymbol{p}=18 \mathrm{KPa}, \boldsymbol{Q}=6.667 \cdot 10^{-5} \mathrm{~m}^{3}
\end{aligned}
$$
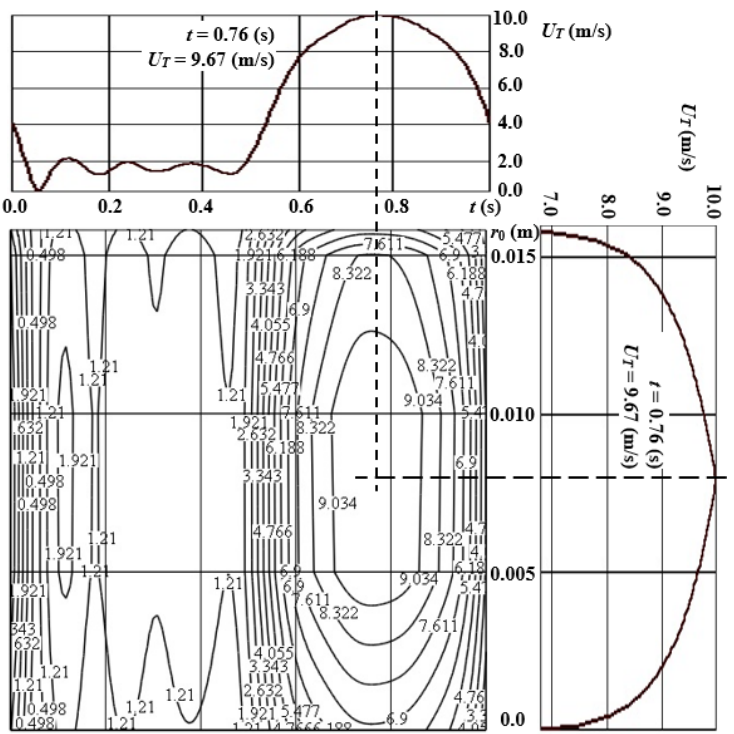

Fig. 2. Representation of change of $\boldsymbol{U}_{\boldsymbol{T}}$ velocity of pulsating flow and $\boldsymbol{U}_{T}$ maximum velocity in $\boldsymbol{t}$ time and over the $\boldsymbol{r}_{\mathbf{0}}$ radius of the hose for $\Delta \boldsymbol{p}=10 \mathrm{KPa}$ pressure

losses and $\boldsymbol{Q}=1.667 \cdot 10^{-5} \mathrm{~m}^{3}$ quantity of liquid corresponding to length of the impulse plug
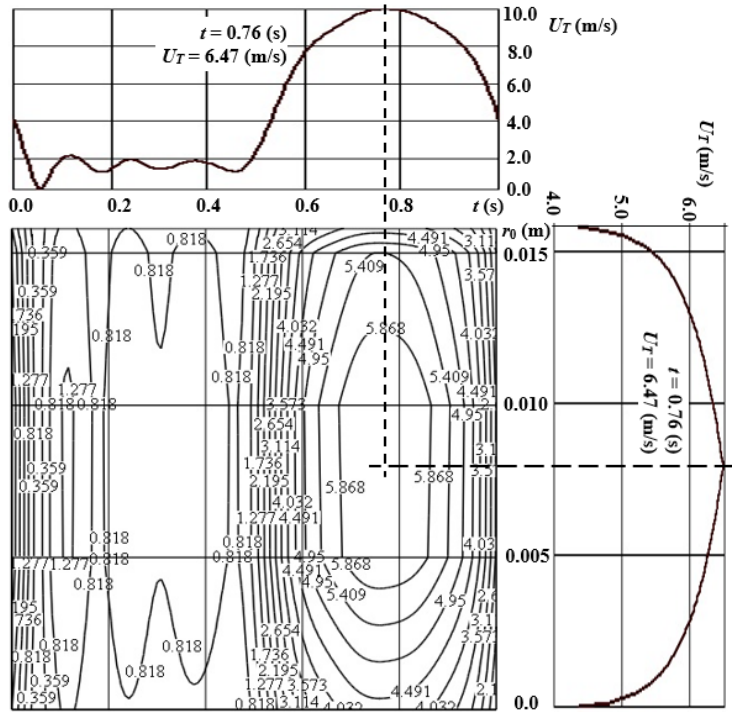

Fig. 3. Representation of change of $\boldsymbol{U}_{T}$ velocity of pulsating flow and $\boldsymbol{U}_{\boldsymbol{T}}$ maximum velocity in $\boldsymbol{t}$ time and over the $\boldsymbol{r}$ o radius of the hose for $\Delta p=18 \mathrm{KPa}$ pressure

losses and $\boldsymbol{Q}=1.667 \cdot 10^{-5} \mathrm{~m}^{3}$ quantity of liquid corresponding to length of the impulse plug

The time of one cycle of flow impulse plug was one second. The pulse character was set as close as possible to the mode of operation of the milking machine pulsator, the frequency was $1 \mathrm{Hertz}$, the offduty factor was ranged from 30 to $70 \%$. The character of the oscillation can be represented as Fourier series or impulse response [19].

The maximum speed is in the centre of the flow. In particular, at $\Delta p=10000 \mathrm{~Pa}$ pressure losses and the $Q=1.667 \cdot 10^{-5} \mathrm{~m}^{3}$ amount of milk flow the pulse rate in the centre of the hose was $U_{T}=9.67 \mathrm{~m} / \mathrm{s}$ on 0.76 second of the cycle of pulse plug of milk flow (Fig. 1a and 2).

At the $\Delta p=18000 \mathrm{~Pa}$ pressure losses and the $Q=1.667 \cdot 10^{-5} \mathrm{~m}^{3}$ amount of milk flow the pulse rate in the centre of the hose was the $U_{T}=6.47 \mathrm{~m} / \mathrm{s}$ on 0.76 second of the cycle of pulse plug of milk flow (Fig. 1, b and 2).

\section{APPLICATION AND INTERPRETATIONS}

In order to understand the use of the model of forced turbulence of a pulsating flow in this section, the application of the results in the diagnosis and measurement of the milk flow velocity by an adaptive milking machine is shown.

As an integral part of cow milking robot, the adaptive milking machine changes its modes according to the flow characteristics of milk. The milk flow is measured at a higher frequency than the frequency of the milking machine pulsator. This is $10 \mathrm{~Hz}$ and above. The milk flow is pulsating and the frequency corresponds to the pulse frequency $-1 \mathrm{~Hz}$. The nature of the milk flow corresponds to the graphical model on Fig. 1. To measure the parameters of milk flow, a thermometer is designed to measure both the velocity and quantity of milk 
[20]. The measured velocity of milk flow corresponds to the real time mode, the time constant of measurement is 10 milliseconds.

The rate of milk ejection by the animal corresponds to the milk flow velocity in the milk hose. The milking machine is adapted by the parameter of the milk flow velocity. Based on information about the quantity of milk per milking cycle and the running value of the vacuum, the processor calculates the milk flow velocity by dependency of (25).

The scheme of the thermoanemometric measuring device for the automated milking machine is shown on Fig. 4 [18].

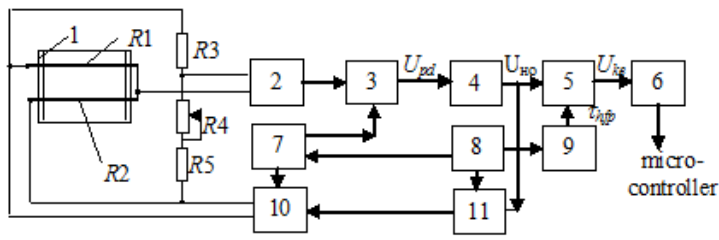

Fig. 4. Block scheme of the thermoanemometric measuring device of the pulsating flow of a two-phase medium: 1 - body; $R 1, R 2$ - thermistor elements; $R 3, R 4$, $R 5$ - resistors of bridge circuit; 2 - alternating current amplifier; 3 - phase detector; 4 - null device;

5 - electronic key; 6 - bus-bar former; 7 - reference generator; 8 - power conditioner; 9 - high frequency pulser; 10 - rectangular oscillation generator,

$$
11 \text { - control unit }
$$

The ratio between the number of pulses (information constituent about milk ejection) at the exit from bus-bar former and the $\left(U_{T}\right)$ milk flow velocity is evaluated by the formula of [18]:

$$
N_{i}=\frac{A \cdot K}{\Delta \tau_{h f p} \cdot\left(P_{H}-P_{o}\right) \cdot S_{s k}} \cdot S_{s k} \cdot \Delta \tau \cdot U_{T},
$$

where $S_{s k} \cdot U_{T} \cdot \Delta \tau=q_{f i}$ - characterizes the milk output for the $\Delta \tau_{i}$ time interval, $\mathrm{m}^{3}$;

$$
A=\frac{5 \cdot R 1^{2} \cdot \Delta R 4 \cdot S_{e t}}{3 \cdot R 3 \cdot \alpha \cdot R 1_{0} \cdot R 2_{0}} ; \quad \Delta R 4=\frac{R 3 \cdot \alpha \cdot R 1_{0} \cdot R 2_{0}}{R 1^{2}} \cdot\left(\Delta t_{2}-\Delta t_{1}\right) ;
$$

$R 1_{0}, R 2_{0}$ - accordingly, the resistance of the thermoresistive elements for a balanced bridge scheme at the $t_{0}$ cooling temperature, $\Omega$;

$t_{1}, t_{2}$ - accordingly, the temperature of heating of $R 1, R 2$ thermoresistive elements, ${ }^{0} \mathrm{C}$;

$\alpha$ - the temperature coefficient of material resistance of the thermoresistive elements, ${ }^{0} \mathrm{C}^{-1}$;

$K$ - the aspect ratio;

$P_{H}$ - the output power of the 10 generator of rectangular oscillations at the amplitude of the output voltage of the heating $U_{H}$ (fig. 5,d), W;

$P_{O}$ - the output power of the generator of rectangular oscillations 10 at the amplitude of the output voltage $U_{O}$ (fig. 5, d) in the mode of "cooling" of the elements, $\mathrm{W}$;

$\Delta \tau=\Delta \tau_{H}+\Delta \tau_{o}$, or (from the fig. $5, \mathrm{c}, \mathrm{d}$ ) $\Delta \tau=\tau_{i}-\tau_{i-1}-$ time interval of one cycle of operation of the circuit (heating-cooling of thermoresistor elements), s;
$\Delta \tau_{H}-$ the duration of heating of thermistor elements during one cycle of automatic operation of the circuit (heating-cooling) (fig. 5, b, d), s;

$\Delta \tau_{0}-$ the duration of "cooling" of the thermistor elements, c;

$S_{s k}$ - the cross-sectional area of milk flow, $\mathrm{m}^{2}$;

$S_{e t}-$ the cross-sectional area of thermoresistive element, $\mathrm{m}^{2}$.

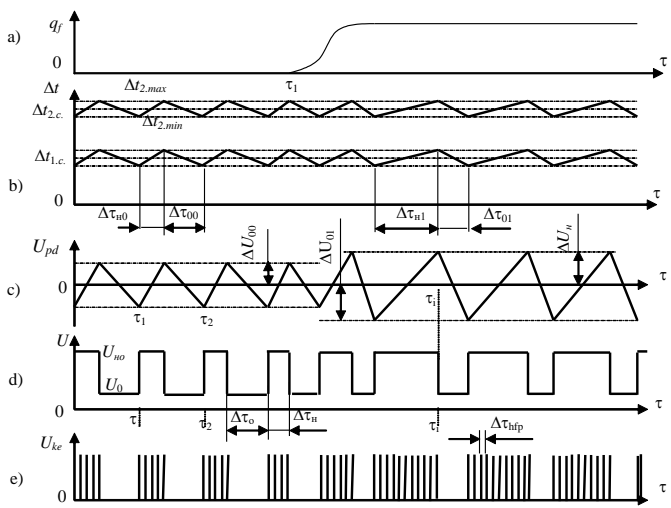

Fig. 5. Work diagrams of thermoanomometric counter of the pulsating flow measuring device of a two-phase medium: a) $q_{n}$ milk ejection; b) $\Delta t_{1}$ and $\Delta t_{2}$ superheat temperature of thermistor elements; c) output voltage of 3 phase detector and input voltage of 4 null device;

d) supply voltage of bridge circuit from the 10 square oscillation generator; e) characteristic of the output voltage of 5 electronic key

The figure 6 represents dependence of the $\boldsymbol{N}_{i}$ number of impulses and the $\boldsymbol{q}$ intensity of milk ejection.

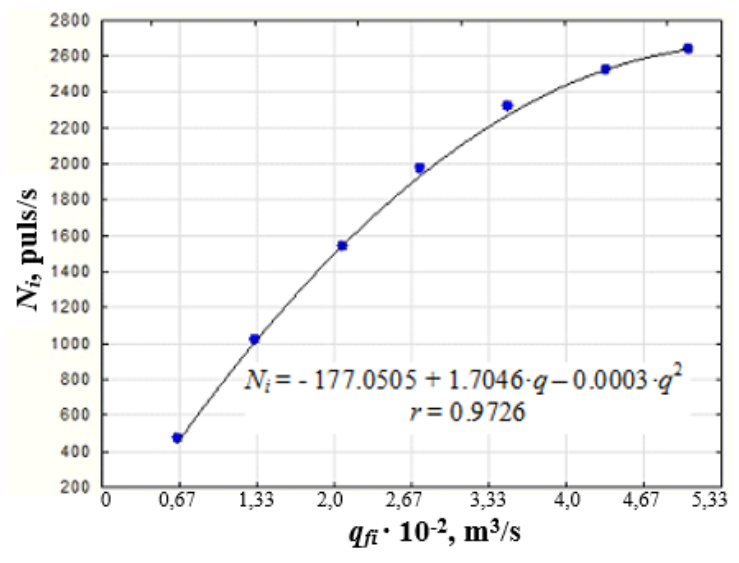

Fig. 6. Dependence of the $\boldsymbol{N}_{\boldsymbol{i}}$ number of impulses of the measurement scheme of the thermoanemometric measuring device on the intensity of $\boldsymbol{q}$ milk ejection

The high-frequency pulse generator operates at the frequency of $12 \mathrm{kHz}$, and the $\Delta \tau_{h f p}=83.3 \mu \mathrm{s}$ accordingly. The time interval of one work cycle of "heating-cooling" of the thermistor elements circuit is $\Delta \tau=1 \mathrm{~s}$.

Knowing the flow velocity, such as milk in a milking machine, under forced turbulence (the mode of motion is pulsating according to the operation of 
the pulsator), after integrating this parameter with a frequency of $100 \mathrm{~Hz}$ and above, the type of milk ejection of an animal is obtained, characterizing the intensity of milk ejection [17]. According to the intensity of animal's milk ejection we can operate by the milking robot or the adaptive milking machine. This is one of the examples of the application an analytical model of the forced turbulence of a pulsating flow for the diagnosis and measurement of kinetic and quantitative characteristics of the flow of complex fluids.

\section{CONCLUSIONS}

The developed analytical dependence of the velocity of the forced turbulence of the pulsating flow allows to calculate the absolute value of the velocity at a given point of cross-section of the pipeline. The deduced representation of (25) equation characterizes the influence of the parameters of the transported medium, dynamic viscosity and density of the medium, dynamic length of pulse plug (due to the amount of medium) and the design parameters of the pipeline (radius) on the velocity impulse. The dynamic characteristics of the flow are taken into account by the pressure drop along the length of the pulse plug of medium flow and by the frequency response and oscillation amplitude of the pulse of pressure drop, which is given by the time equation of oscillation.

The boundary layer is taken into account by the Karman and Landau constants.

Analysis of the results of velocity modelling showed that as the amount of transported medium increases, the flow velocity decreases. The pattern of the velocity change in cross-sectional diameter does not have pronounced parabolic distribution law. In the tangent plane with the boundary layer, the pulsating flow rate is lower by $30 \%$ than the maximum velocity in the centre of the pipeline.

As the dynamic viscosity increased, the velocity of the transported medium decreased.

The developed dependence describes the physical process of Newtonian fluid and gas flows in a pipeline.

This analytical dependence describes the physical process of hydrodynamics of forced turbulence from the standpoint of applied mechanics for Newtonian mediums. Further theoretical and experimental studies of diagnostics of the forced turbulence process for complex Newtonian mediums and the application of developed methods and technical systems of diagnostic will be published.

\section{REFERENCES}

1. Lee TS, Liu X, Li GC, Low HT. Numerical study on sinusoidal fluctuated pulsatile laminar flow through various constrictions. Communications in computational physics. 2007; 2(1): 99-122.
2. Eckhardt B, Schneider TM, Hof B, Westerweel J. Turbulence transition in pipe flow. Annu. Rev. Fluid Mech. 2007; 39: 447-468.

3. Avila K, Moxey D, de Lozar A, Avila M, Barkley D. $\&$ Hof B. The onset of turbulence in pipe flow. Science. 2011; 333 (6039): 192-196.

4. Barkley D, Song BF, Mukund V, Lemoult G, Avila M, Hof B. The rise of fully turbulent flow. Nature. 2015; 526 (7574): 550-553. https://doi.org/10.1038/nature15701

5. Duo $\mathrm{Xu}$, Sascha Warnecke, Baofang Song, Xingyu Ma, Björn Hof. Transition to turbulence in pulsating pipe flow. Journal of Fluid Mechanics. 2017; 831: 418-432. https://doi.org/10.1017/jfm.2017.620.

6. Joel Sundstrom LR, Cervantes MJ. On the similarity of pulsating and accelerating turbulent pipe flows. Flow, Turbulence and Combustion. 2018; 100(2): 417-436. https://doi.org/10.1007/s10494-017-9855-5.

7. Manna M, Vacca A, Verzicco R. Pulsating pipe flow with large-amplitude oscillations in the very high frequency regime. Part 1. Time-averaged analysis. J. Fluid Mech. 2012; 700:246-282. https://doi.org/10.1017/jfm.2012.129

8. Mao Z, Hanratty TJ. Studies of the wall shear stress in a turbulent pulsating pipe flow. J. Fluid Mech. 1986; 170: 545-564.

9. Tardu SF, Binder G, Blackwelder RF. Turbulent channel flow with large-amplitude velocity oscillations. J. Fluid Mech. 1994; 267: 109-151.

10. He S, Jackson JD. An experimental study of pulsating turbulent flow in a pipe. Eur. J. Mech. (B/Fluids). 2009; 28: 309-320.

11. Scotti A, Piomelli U. Numerical simulation of pulsating turbulent channel flow. Phys. Fluids. 2001; 13: 1367-1384.

12. He S, Seddighi M. Transition of transient channel flow after a change in Reynolds number. J. Fluid Mech. 2015; 764:395-427. https://doi.org/10.1017/jfm.2014.698

13. Weng C, Boij S, Hanifi A. Numerical and theoretical investigation of pulsatile turbulent channel flow. J. Fluid Mech. 2016;792:98-133. https://doi.org/10.1017/jfm.2016.73

14. Seddighi M, He S, Vardy AE, Orlandi P. Direct numerical simulation of an accelerating channel flow. Flow Turbul. Combust. 2014; 92: 473-502. https://doi.org/10.1007/s10494-013-9519-z.

15. He S, Jackson JD. A study of turbulence under conditions of transient flow in a pipe. J. Fluid Mech. 2000; 408: 1-38.

16. Dmytriv V, Tkachyshyn R. Investigation of the intensity of milk yield in different modes of operation of milking machines. Bulletin of Lviv State Agrarian University: Agroengineering Research. 2006; 10: 226230.

17. Dmytriv VT. Dynamic modeling of speed ability of milk movement in milking hose of milking machine. Third International Scientific Conference "Measurement, Control and Diagnostics in Technical Systems". Vinnytsia, Ukraine, Vinnytsia National Technical University, October 27-29. 2015; 113-115.

18. Povkh IL. Technical hydrodynamics. Leningrad, Russia: Mashinostroenie, 1976.

19. Dmytriv VT, Dmytriv IV, Lavryk YM. Study of the pressure regulator work with a spring-damper system applied to milking machine, INMATEH - Agricultural Engineering. Bucharest/Romania. 2017; 52(2): 61-67. 
http://www.inmateh.eu/INMATEH_2_2017/52-09-

Dmytriv.pdf

20. Dmytriv V, Dmytriv I, Dmytriv T. Recearch in thermo-anemometric measuring device of pulse flow of two-phase medium. $17^{\text {th }}$ International Scientific Conference: Engineering for rural development. Jelgava, Latvia, University of Life Sciences and Technologies Faculty of Engineering. Proceedings, May 23-25. 2018; 17: 898-904.

https://doi.org/:10.22616/ERDev2018.17.N200

\section{Received 2020-01-07}

Accepted 2020-03-03

Available online 2020-03-10

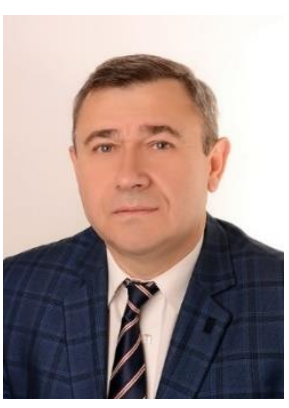

Vasyl DMYTRIV, Professor, Doctor of Technical Sciences (DSc. Eng.), Professor of Department of Mechanics and Automation of Mechanical Engineering, Lviv Polytechnic National University, Ukraine. In scientific work conducts research in the dynamics, synthesis and optimization

hydropneumodynamic, vibration and adaptive cyber-physical systems of automated production processes. Engaged in problems of dynamics, strength and technical diagnostics of machines. Author and co-author of more than 350 scientific publications, including 7 monographs, 14 manuals and 36 patents. 